\title{
Use of a novel extended blink test to evaluate the performance of two polyvinylpyrrolidone- containing, silicone hydrogel contact lenses
}

This article was published in the following Dove Press journal:

Clinical Ophthalmology

\section{Jeffery Schafer' \\ William Reindel' \\ Robert Steffen' \\ Gary Mosehauer' \\ Joseph Chinn²}

'Vision Care, Bausch and Lomb Incorporated, Rochester, NY, USA;

${ }^{2} \mathrm{~J}$ Chinn LLC, Lafayette, CO, USA
Correspondence: William Reindel Vision Care, Bausch and Lomb Incorporated, 1400 N Goodman St. Rochester, NY 14609, USA

Tel +I 5853386000

Email bill.reindel@bausch.com
Background: Sustained digital display viewing reduces eye blink frequency and tear film stability. To retain water and preserve a smooth optical surface, contact lens manufacturers have integrated the humectant polyvinylpyrrolidone (PVP) into silicone hydrogel contact lenses. In this study, extended blink time (EBT) was used to assess visual stability over a prolonged blink interval of two PVP-containing silicone hydrogel lenses, samfilcon A (SAM) and senofilcon A (SEN).

Materials and methods: This randomized, bilateral, masked, crossover study assessed lens performance in ten subjects after 16 hours of wear. EBT, ie, the time elapsed between cessation of blinking and blur-out of a threshold letter on the acuity chart, was measured. At the end of the wear period, subjects reported duration of computer use and rated visual quality (VQ) and comfort while wearing the assigned lens, and the investigator evaluated lens surface wetting characteristics. Each lens was removed and immediately weighed to determine total water content.

Results: EBTs were 10.42 seconds for SAM and 8.04 seconds for SEN ( $p=0.015)$. Subjective ratings of VQ after 16 hours of wear were 84.6 for SAM and 74.4 for SEN ( $p=0.049)$. Comfort ratings were 85.9 for SAM and 80.2 for SEN $(p>0.05)$. Median times of computer use were 6-8 hours for both lens types. Post blink, $70.0 \%$ of SAM and $30.0 \%$ of SEN lenses were completely wet $(p=0.021)$. Total water content after wear was $43.7 \%$ for SAM and $35.5 \%$ for $\operatorname{SEN}(p<0.001)$.

Conclusion: EBT measurement indicated more stable vision with the PVP-containing SAM polymer compared with the PVP-containing SEN polymer. The SAM polymer also demonstrated better surface wetting and maintained higher water content after a prolonged period of wear. EBT can be valuable in assessing vision stability of patients after hours of computer use.

Keywords: polyvinylpyrrolidone, PVP, digital video display, extended blink time, EBT, visual quality, VQ, lens hydration

\section{Introduction}

Contact lens wearers often report increased dryness and discomfort, as well as tired eyes toward the end of the day. ${ }^{1}$ By contrast, symptoms of blurry or fluctuating vision often start in the morning and persist throughout the day. ${ }^{2}$ The latter complication can be exacerbated by the use of the digital display devices pervasive in today's society. From smart phones to tablets to televisions to personal computers, Americans spend over 10 hours viewing electronic device displays daily.,4

The widespread use of digital displays represents a challenge for contact lens makers to design and manufacture contact lenses that perform well under conditions of 
prolonged digital display viewing. ${ }^{5}$ The consequences of such prolonged digital display viewing upon both visual acuity and comfort were recently described. ${ }^{6}$ Briefly, the digital display environment results in decreased blink frequency, increased tear evaporation, incomplete blinking, decreased tear stability, and tear film disruption in both naked eyes ${ }^{7-10}$ and the eyes of the contact lens wearer ${ }^{11,12}$ alike. In the latter case, this manifests as blurry or fluctuating vision that may indicate a lens surface not retaining water. ${ }^{13}$ The need for the development of new materials that better retain water becomes greater as the number of different digital devices used and daily screen viewing time increase.

Similarly, contact lens wear is also identified as a risk factor in computer vision syndrome (CVS), a combination of eye and vision problems associated with the use of computers ${ }^{14,15}$ and characterized by symptoms including neck/shoulder/back pain, eye strain, headache, blurred vision, and dry eyes. ${ }^{16}$ Mechanisms contributing to CVS include decreased blink frequency and completeness of blink, increased tear evaporation rate, and decreased tear film stability. ${ }^{9,13}$ Commonalities in symptoms and mechanisms suggest that digital eye strain and CVS are related if not the same, with the distinction that digital devices are typically viewed at closer distance than are computer monitors.

In both digital eye strain and CVS, blink rate and blink characteristics change during prolonged periods of concentration. ${ }^{17}$ For example, blink rate in normal, nonlens-wearing subjects decreased from nominal 17 blinks per minute at rest down to 4.5 blinks per minute when reading, ${ }^{18}$ and from $18 \pm 5.7$ blinks per minute down to $3.6 \pm 1.8$ blinks per minute during video display viewing. ${ }^{7}$ It should be noted that blink frequency varies widely, both within a study due to patient factors and between studies due to differences in specific study conditions. In a more recent study conducted after the advent of smart phones, blink rate in adapted habitual contact lens-wearing subjects similarly decreased from 19 blinks per minute while listening to music down to 6.2 blinks per minute while playing a video game when not wearing lenses. ${ }^{12}$ The same subjects' blink rate decreased from 27 blinks per minute down to 23 blinks per minute when wearing lenses, although this was not statistically significant. Under both conditions, subjects blinked less while playing the video game (blink amplitude [BA], defined as the maximum percentage closure of the palpebral aperture during one blink cycle, of 77 while listening to music versus 67 during game play when not wearing lenses; BA of 84 while listening to music versus 70 during game play when wearing lenses). Incomplete blinking during computer use was similarly reported and found to have greater effect upon CVS symptoms, ${ }^{19}$ as tear breakup and evaporation during incomplete blinking contribute to lens water loss. ${ }^{20}$

Soft contact lenses tend to lose water to some extent during wear. ${ }^{21}$ Dehydration of high water lenses and concomitant reduction in visual acuity subsequent to reduced blinking or a drafty environment was reported three decades ago and termed as dehydration blur, ${ }^{22}$ yet more recently the phenomenon has been reported to occur with low-water, silicone hydrogel lenses as well. ${ }^{23}$ While lenses of lower water content have been reported to dehydrate less than those of higher water content, and silicone hydrogels (typically of lower water content) less than traditional hydrogels ${ }^{21,24}$ (typically of higher water content), ${ }^{25}$ recent advances in material and surface chemistry resulted in newer high water lenses such as nesofilcon A that does not follow this trend. ${ }^{6}$ Further studies to advance understanding of the chemical and surface properties of lens polymers that maintain water in both low and high water traditional hydrogel and silicone hydrogel lenses will drive development of future lens generations.

The ramifications of contact lens dehydration upon both visual acuity and comfort were recently described. ${ }^{6}$ Briefly, lens dehydration both disrupts the lens tear film ${ }^{26}$ and effects subtle changes in lens dimension; ${ }^{27}$ these phenomena lead to light scatter and visual aberration, ${ }^{13,28,29}$ as well as contact lens-induced dry eye. ${ }^{30}$

One strategy that lens manufacturers use to improve contact lens water retention and preserve a smooth optical surface is to integrate wetting agents into lens polymers. ${ }^{31}$ One such agent is Pluronic ${ }^{\circledR}$ F127 (BASF, Florham Park, NJ, USA), a polyethylene oxide (PEO)-polypropylene oxidePEO block copolymer surfactant, which is incorporated into nesofilcon $\mathrm{A}^{32}$ (Bausch and Lomb Incorporated, Rochester, NY, USA), a US Food and Drug Administration (FDA) Group II (high water content, nonionic) hydrogel material. ${ }^{33}$ This lens was reported to maintain greater than $98 \%$ of its initial water over the course of a 16 -hour wearing day; ${ }^{34}$ as such, the lens maintains a better predicted retinal image quality over time compared with etafilcon A based upon an in vitro method to predict $\log$ MAR visual acuity. ${ }^{35}$ Another such agent is polyvinylpyrrolidone (PVP) that has proven to be highly effective, as lenses incorporating it into the polymer matrix have found both clinical and commercial success. ${ }^{36}$ N-vinyl pyrrolidone (NVP), the monomer from which PVP is formed, was used as a co-monomer in several commercial contact lens materials over the past two decades due to the water-attracting properties of the resultant polymers. 
Fully formed PVP was first incorporated into commercial silicone hydrogel lens materials as an additive in the early 2000s (galyfilcon $\mathrm{A}^{37}$ and senofilcon $\mathrm{A}[\mathrm{SEN}],{ }^{38}$ both from Vistakon Division of Johnson and Johnson Vision Care, Jacksonville, FL, USA). Recently, samfilcon A (SAM) ${ }^{39}$ (Bausch and Lomb Incorporated) integrated the PVP wetting agent into the polymer matrix through a novel manufacturing process that results in PVP tightly compacted at the surface. ${ }^{40}$ A PVP-rich surface can create an environment that attracts and retains water, resulting in a highly wettable and smooth surface.

While both polymers include PVP as an internal wetting agent, the lenses differ in their fabrication methods and relative amounts of PVP. In the manufacture of the SEN lens, fully formed high molecular weight PVP is added to the monomer mixture prior to synthesis. In the manufacture of the SAM lens, the silicone monomers and NVP polymerize sequentially, first forming the silicone hydrogel matrix and then forming PVP, the latter present in fourfold greater quantity compared with SEN. ${ }^{41}$ While similar in chemistry, SEN and SAM lenses have been characterized as silicone surrounding PVP and PVP surrounding silicone, respectively. ${ }^{40}$

Even with reports in published literature of subjective comfort while wearing both SEN and SAM lenses, assessment of visual stability is lacking. Because water loss increases as blinks become less frequent while viewing a video display, ${ }^{29}$ it is of interest to know how PVP internal wetting agents affect not only lens water retention and comfort under these conditions, but also visual quality (VQ). Therefore, an extended blink test was developed, and SEN and SAM silicone hydrogel lenses that incorporate PVP were compared with respect to dehydration resistance, open eye visual stability, VQ, comfort, and lens wetting over a prolonged blink interval.

\section{Materials and methods}

This study was reviewed and approved by Southwest Independent Institutional Review Board (Fort Worth, TX, USA). Written approval of the protocol, informed consent form, and subject instructions were provided prior to initiation of the study. Eligible subjects gave written informed consent and complied with the study procedures.

\section{Lenses}

SEN (38\% water content), an FDA Group V-Cr ${ }^{33}$ (low water content, nonionic, non-surface treated, semi-interpenetrating network) silicone hydrogel lens, and SAM (46\% water content), an FDA Group V-C $\mathrm{C}^{33}$ (low water content, nonionic, hydrophilic monomer) silicone hydrogel lens, were tested and compared.

\section{Lens water content determination}

Lens water content was determined by a modification of the method outlined in the International Organization for Standardization standard. ${ }^{42}$ Water content was measured and calculated for individual lenses rather than pooled lenses specified in the standard. This allowed for the calculation of water loss pursuant to lens wear for individual lenses.

\section{Clinical evaluation}

A total of 10 healthy current (9) and former (1) contact lenswearing subjects participated in this randomized, "doublemasked" evaluation. One subject was a former lens wearer who had reverted to eyeglasses, while another subject wore no lens in one eye and a nesofilcon A lens in the contralateral eye. All other subjects wore the same lens in each eye. Subjects' habitual lenses are summarized in Table 1; no subject wore either test lens habitually. Each subject wore both test lens types in randomized order, ie, subjects wore either SAM or SEN lenses in both eyes over a 16-hour wear period on the first test day and then the other lens type on a second test day. All subjects were asked how much time they spent working with a computer on the day of each test. Although all were coincidentally habitual computer users, this was not a study inclusion or exclusion criterion. All clinical and nonclinical assessments were made at the end of the wear period. While investigators did not intentionally view the manufacturer's identifying markings on either lens, unconscious practitioner bias remains possible.

Both objective and complementary subjective evaluations of lens performance were used in this study. A pilot extended blink test was developed to assess visual stability over a prolonged blink interval. In this test, the time elapsed between cessation of blinking and blur-out of a threshold letter on the

Table I Habitual lenses of test subjects

\begin{tabular}{llll}
\hline Material & Brand name & Manufacturer & $\mathbf{n}$ \\
\hline No lens & & & $3^{*}$ \\
Lotrafilcon B & Air Optix Night and Day & $\begin{array}{l}\text { Alcon laboratories, Inc } \\
\text { (Ft Worth, TX, USA) }\end{array}$ & 2 \\
Balafilcon A & PureVision2 & Bausch and Lomb & 2 \\
& PureVision Multifocal/HA & Incorporated & 2 \\
& PureVision Multifocal/LA & (Rochester, NY, USA) & 2 \\
Hilafilcon B & SofLens Daily Disposable & & 2 \\
Nesofilcon A & Biotrue ONEday & & $7^{*}$ \\
\hline
\end{tabular}

Notes: *One subject was a former lens wearer who had reverted to eyeglasses, while another subject wore no lens in one eye and a Biotrue ONEday lens in the contralateral eye. All other subjects wore the same lens in each eye. $\mathrm{n}=$ number of eyes. 
acuity chart was measured in seconds. The investigator also evaluated lens fit and surface wetting characteristics with normal blinking using a slit lamp. Wetting extent was rated in one of five categories: 1) absence of nonwetting areas, 2) $<8 \%$ of surface area nonwetting, 3 ) $<17 \%$ of surface area nonwetting, 4) $<25 \%$ of surface area nonwetting, and 5) $>25 \%$ of surface area nonwetting. At the end of the evaluation, lenses were removed and immediately weighed to determine total water content, and lens types were compared.

Subjects were asked to rate both their VQ and comfort at the end of the 16-hour wear period on a 0 to 100 scale, with a score of 100 considered the best. Each subject scored each lens in each eye individually. Subjects also reported the amount of time they spent at a computer while wearing the study lenses.

\section{Statistical analysis}

Extended blink time (EBT), subjective VQ, and subjective comfort ratings were averaged over each subject's two eyes prior to analysis. The differences between lenses in these outcomes were tested for significance using linear mixedeffects models including the fixed effects of lens type and crossover phase and the random effect of subject. The difference between lenses in water content was tested for significance using a two-sided, one-sample $t$-test. The difference between lenses in the fraction of lenses completely wettable was tested for significance using McNemar's test. Differences were considered significant if $p \leq 0.05$.

\section{Results}

Subjects reported comparable total duration of computer use, typically 6-8 hours for both lens types (Table 2).

Performance characteristics of the tested lenses are reported in Table 3. The SAM lenses demonstrated better visual stability than did the SEN lenses, with longer elapsed time until blur during the extended blink test $(10.42 \pm 4.86$ seconds for SAM versus $8.04 \pm 2.69$ for SEN, $p=0.015$ ). The SAM lenses also demonstrated better subjective VQ at the end of the wear period than did the SEN lenses (VQ rating of $84.6 \pm 16.8$ for SAM versus $74.4 \pm 19.8$ for SEN, $p=0.049$ ).

Table 2 Self-reported duration of computer use $(n=10$ subjects for each lens type)

\begin{tabular}{|c|c|c|}
\hline Lens & Samfilcon A & Senofilcon A \\
\hline Time, $\mathbf{h}$ & \multicolumn{2}{|c|}{ Frequency, \% } \\
\hline $\mathrm{I}-2$ & 0 & 0 \\
\hline $3-5$ & 30 & 20 \\
\hline $6-8$ & 60 & 70 \\
\hline 9 & 10 & 10 \\
\hline
\end{tabular}

Table 3 Lens performance characteristics after 16 hours of wear ( $\mathrm{n}=10$ subjects for each lens type)

\begin{tabular}{|c|c|c|c|c|c|}
\hline \multirow{2}{*}{$\frac{\text { Lens }}{\text { Variable }}$} & \multicolumn{2}{|c|}{ Samfilcon A } & \multicolumn{2}{|c|}{ Senofilcon A } & \multirow[t]{2}{*}{$p$-value } \\
\hline & Mean & sdev & Mean & sdev & \\
\hline EBT, seconds & 10.42 & 4.86 & 8.04 & 2.69 & 0.015 \\
\hline Visual quality & 84.6 & 16.8 & 74.4 & 19.8 & 0.049 \\
\hline Comfort rating & 85.9 & 24.4 & 80.2 & 21.6 & 0.40 \\
\hline
\end{tabular}

Abbreviation: EBT, extended blink time.

Although not significantly different, comfort ratings also favored the SAM lenses (comfort rating of $85.9 \pm 24.4$ for SAM versus $80.2 \pm 21.6$ for SEN, $p=0.40$ ).

On examination with a slit lamp at the end of the 16-hour wear period and just before lens removal, $70.0 \%$ of the SAM lenses demonstrated a perfectly wettable surface (absence of any nonwetting areas) post blink, compared with only $30.0 \%$ of SEN lenses $(p=0.021)$. Just after lens removal, total water content was $43.7 \% \pm 0.7 \%$ for SAM lenses and $35.5 \% \pm 1.1 \%$ for SEN lenses $(p<0.001)$.

\section{Discussion}

Pervasive use of more digital devices for longer durations has greatly increased the incidence of symptoms of dryness, tired eyes, eye health, and vision problems, ${ }^{43}$ due to primarily decreased blink frequency and incomplete blinking leading to tear film disruption during video display use..$^{7-12}$ This becomes especially problematic during contact lens wear and establishes the need for development of contact lenses that better maintain lens water and a more stable tear film.

Contact lens-wearing patient perceptions also illustrate the need for such lenses. A recent survey of 568 subjects (136 daily disposable and 432 planned replacement contact lens wearers) found high incidences of wearers concerned about blurry/fluctuating vision (56\%), bothered by blurry/ fluctuating vision (68\%), and that found blurry/fluctuating vision to have a high negative impact on lens wearing experience $(70 \%))^{2}$ Blurry/fluctuating vision concerned subjects more than did any other negative symptom. To cope with this symptom, subjects typically blink more frequently. However, this reflex is suppressed during periods of intense concentration such as when viewing digital video displays. ${ }^{17}$

When 287 of the original test subjects were specifically asked to track symptoms of dry eyes, tired eyes, and blurry/ fluctuating vision throughout the day, the reported incidence of these symptoms increased. In the morning (when many are engaged in computer work), the reported incidence of blurry/ fluctuating vision (35\%) was greater than the reported incidences of both dry eyes (16\%) and tired eyes $(6 \%)$ (Figure 1$){ }^{2}$ By evening, the reported incidence of tired eyes (64\%) was 


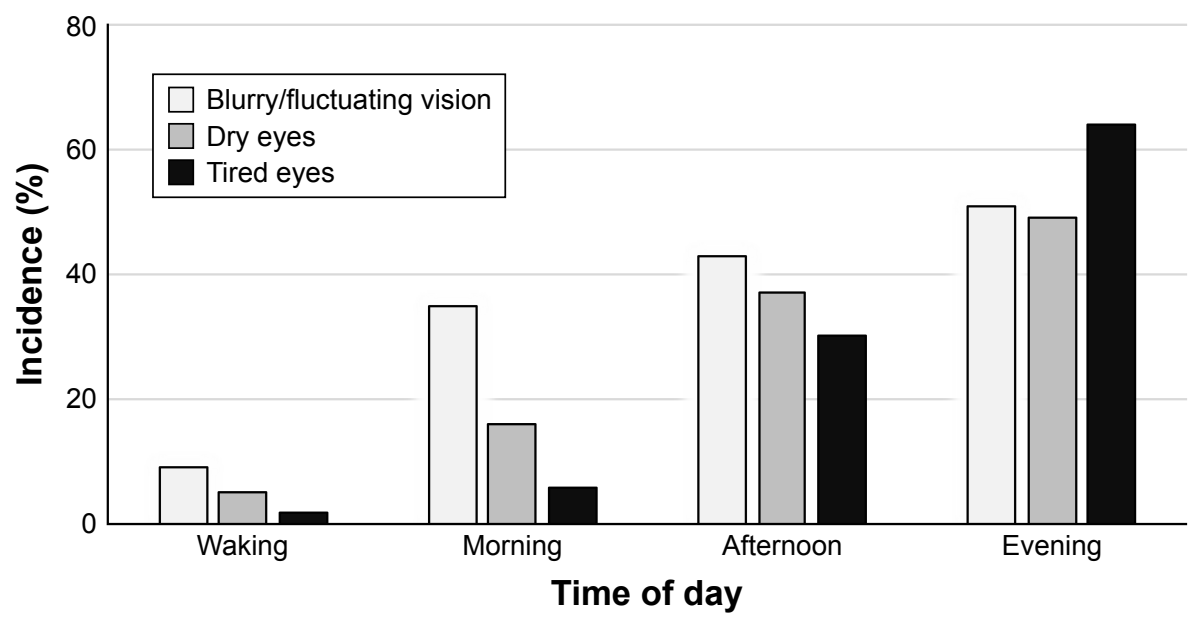

Figure I Incidence of reported adverse symptoms of blurry/fluctuating vision, dry eyes, and tired eyes throughout the wearing day.

Notes: Copyright @2016. Bausch and Lomb Incorporated. Reproduced from Epstein AB, Wilson B, Reindel WT. How visual performance influences patients' perceptions of contact lens wear. CL Spectrum. 2016;3I(I3):20-25. ${ }^{2}$

greater than the incidences of both blurry/fluctuating vision (51\%) and dry eyes (49\%).

In a previous study, a novel in vitro method predicted $\log$ MAR score based on optical image quality as the lens dehydrates with time. The study demonstrated that the SAM lens achieved better predicted logMAR scores at time zero and over the full 30s study time course, with 1.3 line mean improvement at the 30 s time point compared with the SEN lens $(p<0.01) .{ }^{44}$ While the measurements provided an objective estimation of image quality, they were not on-eye and did not provide a true measure of vision loss.

In the present clinical study, the assessment provided an on-eye measure of vision loss. EBTs while wearing SEN or SAM lenses were measured and compared to determine the relative effectiveness of the PVP wetting agents to maintain water and optical clarity in between the extended blinks that occur during digital display viewing. The measured EBT of the test subjects was $30 \%$ greater when wearing SAM lenses than when wearing SEN lenses $(10.42 \pm 4.86$ versus $8.04 \pm 2.69$, respectively; $p=0.015$, Table 3 ).

The EBT variable differs from the noninvasive tear film breakup time, which is the time between the end of a complete blink and the first appearance of breaks in a reflected grid pattern without respect to visual acuity changes prior to tear film breakup. ${ }^{45}$ Torkildsen used a similar test (Inter-blink Interval Visual Acuity Decay) to evaluate the ability of two lubricant eye drops to delay loss of visual function in dry eye patients. ${ }^{46}$ EBT offers greater insight into vision as it is the time between the end of a complete blink and first loss of visual acuity as the lens surface dehydrates on the open eye. LogMAR charts used for objective evaluation of contact lens visual performance ${ }^{47}$ prove useful in this regard as adapted in the present study to capture the kinetics of vision change during restricted blinking. The greater resilience in stable vision may be due to the higher PVP content and specific character of PVP at the surface of SAM.

At the end of the wear period, the VQ of the SAM lens was rated significantly higher than that of the SEN lens $(84.6 \pm 16.8$ versus $74.4 \pm 19.8 ; p=0.049)$. The time course and mechanism of deterioration of optical quality and VQ upon tear breakup while wearing silicone hydrogel lenses and suppressing blinking indicate that the breakup of the tear film introduces refractive aberrations and reflective distortions from the interface between the air and tear film. With the optical changes, the contrast sensitivity decreases with the decrease in image quality. ${ }^{48}$

Overall, SAM lenses maintained more water than did SEN lenses at the time of removal $(43.7 \% \pm 0.7 \%$ versus $35.5 \% \pm 1.1 \% ; p<0.001)$. More water associated with higher PVP content and specific character of PVP at the surface of SAM may also help to explain differences in the assessment of surface wettability. After 16 hours of wear, significantly more SAM lenses had $100 \%$ wettable surfaces $(70.0 \%$ versus $30.0 \% ; p=0.021)$. Loss of wetting at the lens surface can alter the local refractive index in areas of nonwetting, and thus introduce refractive aberrations that impact VQ.

Tear film instability has also been linked to hyperosmolarity, and it is suggested that the instability may result in transient spikes in osmolarity that lead to triggering of sensory neurons. ${ }^{49}$ Although not significantly different, comfort ratings also favored the SAM over the SEN lens $(85.9 \pm 24.4$ versus $80.2 \pm 21.6 ; p=0.40$ ). A larger sample size would be required to determine if apparent greater comfort while wearing SAM lenses becomes significant. Further research into the role of material characteristics, tear film instability, hyperosmolarity, and discomfort is warranted. 
Wetting agents are used in both traditional and silicone hydrogel lenses to promote water retention and inhibit lens dehydration, ${ }^{31}$ but their effects upon visual stability are sparsely reported in published literature. Materials reported effective in promoting water retention include polyethylene glycol, ${ }^{50}$ hyaluronic acid, ${ }^{51}$ phosphorylcholine, ${ }^{52}$ and PVP. ${ }^{53}$ Clinically, PVP wetting agent incorporated into one contact lens material resulted in improved optical quality and reduced dryness relative to the same material without PVP after 1 hour of lens wear in symptomatic patients..$^{53}$ Although the present study was not designed to separate the effects of individual lens characteristics such as the wetting agent, the lens containing more PVP (SAM) outperformed the lens containing less PVP (SEN) with respect to maintenance of visual acuity during suppressed blink after 16 hours of wear. While both PVP-containing lenses were similar in comfort rating, digital device display-viewing wearers of SAM lenses might experience better vision than wearers of SEN lenses. This is significant because inhibited blinking as occurs during digital display viewing can result in a poor lens wearing experience and subsequent discontinuation of lens wear. ${ }^{26}$

\section{Conclusion}

The extended blink test developed for this study demonstrated more stable vision with the PVP-containing SAM polymer compared to the PVP-containing SEN polymer (EBT = $10.42 \pm 4.86$ seconds versus $8.04 \pm 2.69$ seconds; $p=0.015$ ). Further, subjects rated VQ better when wearing SAM lenses than when wearing SEN lenses (VQ rating of $84.6 \pm 16.8$ versus $74.4 \pm 19.8 ; p=0.049)$. SAM lenses maintained greater water content than did SEN lenses $(43.7 \% \pm 0.7 \%$ versus $35.5 \% \pm 1.1 \% ; p<0.001)$. Better visual stability and higher water content reflected as a higher percentage of completely wetted SAM lenses compared with SEN lenses $(70.0 \%$ versus $30.0 \% ; p=0.021)$. A trend toward greater overall comfort with SAM lenses was observed, but the difference was not significant (comfort rating of $85.9 \pm 24.4$ versus $80.2 \pm 21$. 6 ; $p=0.40$ ). While the extended blink test has not yet been rigorously validated, it nonetheless can be valuable in the development of lens polymers to meet today's visual demands, as well as in the assessment of visual stability of patients after hours of computer use and other modes of digital device viewing.

\section{Acknowledgments}

This work was funded by Bausch and Lomb Incorporated. The abstract of this paper was presented at the American Academy of Optometry meeting held at Anaheim, CA, USA, on November 9-12, 2016, as a poster presentation (program number 165121) with interim findings. The poster's abstract was published online and is available for download at https:// www.aaopt.org/detail/knowledge-base-article/use-novelextended-blink-test-evaluate-polyvinylpyrrolidone-pvpcontact-lens-containing-polymer.

\section{Disclosure}

Joseph Chinn is a paid consultant of Bausch and Lomb Incorporated. All other authors are employees of Bausch and Lomb Incorporated. The authors report no other conflicts of interest in this work.

\section{References}

1. Fonn D. Targeting contact lens induced dryness and discomfort: what properties will make lenses more comfortable. Optom Vis Sci. 2007; 84(4):279-285.

2. Epstein AB, Wilson B, Reindel WT. How visual performance influences patients' perceptions of contact lens wear. CL Spectrum. 2016; 31(13):20-25.

3. Nielsen.com [homepage on the Internet]. The Total Audience Report: Q1 2016. New York, NY: Nielsen Holdings PLC; 2016 [updated June 27, 2016; cited September 1, 2017]. Available from: http://www.nielsen. com/us/en/insights/reports/2016/the-total-audience-report-q1-2016. html. Accessed March 14, 2018.

4. Ipsos.com [homepage on the Internet]. Socialogue: If you're awake, chances are you are well-connected. New York: Ipsos OTX and Ipsos Global@dvisor; 2015 [updated August 21, 2012; cited September 1, 2017]. Available from: https://www.ipsos.com/en-us/socialogue-if-youreawake-chances-are-you-are-well-connected. Accessed March 14, 2018.

5. Rosenfield M, Howarth PA, Sheedy JE, Crossland MD. Vision and IT displays: a whole new visual world. Ophthalmic Physiol Opt. 2012; 32(5):363-366.

6. Schafer J, Steffen R, Reindel W, Chinn J. Evaluation of surface water characteristics of novel daily disposable contact lens materials, using refractive index shifts after wear. Clin Ophthalmol. 2015;9:1973-1979.

7. Patel S, Henderson R, Bradley L, Galloway B, Hunter L. Effect of visual display unit use on blink rate and tear stability. Optom Vis Sci. 1991; 68(11):888-892.

8. Tsubota K, Nakamori K. Dry eyes and video display terminals. $N$ Engl J Med. 1993;328(8):584.

9. Cardona G, García C, Serés C, Vilaseca M, Gispets J. Blink rate, blink amplitude, and tear film integrity during dynamic visual display terminal tasks. Curr Eye Res. 2011;36(3):190-197.

10. Himebaugh NL, Begley CG, Bradley A, Wilkinson JA. Blinking and tear break-up during four visual tasks. Optom Vis Sci. 2009;86(2):E106-E114.

11. Kojima T, Ibrahim OM, Wakamatsu T, et al. The impact of contact lens wear and visual display terminal work on ocular surface and tear functions in office workers. Am J Ophthalmol. 2011;152(6):933-940.

12. Jansen ME, Begley CG, Himebaugh NH, Port NL. Effect of contact lens wear and a near task on tear film break-up. Optom Vis Sci. 2010;87(5): 350-357.

13. Wolkoff P, Nøjgaard JK, Troiano P, Piccoli B. Eye complaints in the office environment: precorneal tear film integrity influenced by eye blinking efficiency. Occup Environ Med. 2005;62(1):4-12.

14. Blehm C, Vishnu S, Khattak A, Mitra S, Yee RW. Computer vision syndrome: a review. Surv Ophthalmol. 2005;50(3):253-262.

15. Rosenfield M. Computer vision syndrome: a review of ocular causes and potential treatments. Ophthalmic Physiol Opt. 2011;31(5):502-515.

16. The Vision Council. Hindsight is 20/20/20: Protect Your Eyes from Digital Devices. 2015 Digital Eye Strain Report. Alexandria, VA: The Vision Council. 2015 [cited September 1, 2017]. Available from: http://www.pcom.ph/sites/default/files/downloads/vc_digitaleyestrain_ report2015.pdf. Accessed March 14, 2018.

17. Gowrisankaran S, Nahar NK, Hayes JR, Sheedy JE. Asthenopia and blink rate under visual and cognitive loads. Optom Vis Sci. 2012;89(1): 97-104. 
18. Bentivoglio AR, Bressman SB, Cassetta E, Carretta D, Tonali P, Albanese A. Analysis of blink rate patterns in normal subjects. Mov Disord. 1997;12(6):1028-1034.

19. Portello JK, Rosenfield M, Chu CA. Blink rate, incomplete blinks and computer vision syndrome. Optom Vis Sci. 2013;90(5):482-487.

20. McMonnies CW. Incomplete blinking: exposure keratopathy, lid wiper epitheliopathy, dry eye, refractive surgery, and dry contact lenses. Cont Lens Anterior Eye. 2007;30(1):37-51.

21. Ramamoorthy P, Sinnott LT, Nichols JJ. Contact lens material characteristics associated with hydrogel lens dehydration. Ophthalmic Physiol Opt. 2010;30(2):160-166.

22. Keeney AH, Shrader EC. Kinetic visual disturbances with contact lenses. Surv Ophthalmol. 1983;28(2):112-116.

23. Hovinga KR, Ludington PD, Merchea M, Steffen R. Preventing dehydration blur. CL Spectrum. 2014;29(13):39-42.

24. Alemany AL, Refojo MF. Comparative study of the hydration of hydrophilic contact lenses by refractive index and gravimetry. CLAOJ. 2000;26(4):200-203.

25. Jones L, May C, Nazar L, Simpson T. In vitro evaluation of the dehydration characteristics of silicone hydrogel and conventional hydrogel contact lens materials. Cont Lens Anterior Eye. 2002;25(3): $147-156$

26. Korb DR. Tear film-contact lens interactions. Adv Exp Med Biol. 1994;350:403-410.

27. Tranoudis I, Efron N. In-eye performance of soft contact lenses made from different materials. Cont Lens Anterior Eye. 2004;27(3):133-148.

28. Timberlake GT, Doane MG, Bertera JH. Short-term, low-contrast visual acuity reduction associated with in vivo contact lens drying. Optom Vis Sci. 1992;69(10):755-760.

29. Tutt R, Bradley A, Begley C, Thibos LN. Optical and visual impact of tear break-up in human eyes. Invest Ophthalmol Vis Sci. 2000;41(13): 4117-4123.

30. Guillon M, Maissa C. Contact lens wear affects tear film evaporation. Eye Contact Lens. 2008;34(6):326-330.

31. Keir N, Jones L. Wettability and silicone hydrogel lenses: a review. Eye Contact Lens. 2013;39(1):100-108.

32. FDA 510(k) Summary K113703. Bausch + Lomb nesofilcon A Contact Lens. Available from: https://www.accessdata.fda.gov/cdrh_docs/ pdf11/K113703.pdf. Accessed March 14, 2018.

33. Fda.gov. FDA Executive Summary. Prepared for the May 13, 2014 Meeting of the Ophthalmic Devices Panel of the Medical Devices Advisory Committee. Contact Lens and Care Product Guidance Documents. Rockville, MD: US Food and Drug Administration [updated June 27, 2016; cited September 1, 2017]. Available from: https://creativesrv.bid/ library/?sid=rwHURXwhuRGCIH8tyL42nQm1kWWMxinLqASe2IM P0MombfhRhGJek51jW516oXPoLIJBAczaplY4npMyhoIHGcC5Bj1 G8dBbNf3v\&fn=FDA \%20Executive $\% 20$ Summary $\% 20$ Prepared $\% 20$ for $\% 20$ the $\% 20$ May $\% 2013, \% 202014 \% 20$ Meeting\%20of.pdf. Accessed March 14, 2018.

34. Steffen R, Schafer J. Comparing on eye dehydration of two hydrogel contact lenses. Poster presented at: Global Specialty Lens Symposium; January 25, 2014; Las Vegas, NV.

35. Lee RH, Kingston AC, Richardson G. Evaluation of contact lens image stability and predicted $\log$ MAR image resolution as lenses dehydrate. Invest Ophthalmol Vis Sci. 2012;53:6110.

36. Jones LW, Subbaraman LN, Rogers R, Dumbleton K. Surface treatment, wetting and modulus of silicone hydrogels. Optician. 2006;232:28-34.

Clinical Ophthalmology

\section{Publish your work in this journal}

Clinical Ophthalmology is an international, peer-reviewed journa covering all subspecialties within ophthalmology. Key topics include: Optometry; Visual science; Pharmacology and drug therapy in eye diseases; Basic Sciences; Primary and Secondary eye care; Patient Safety and Quality of Care Improvements. This journal is indexed on
37. FDA 510(k) Summary of Safety and Effectiveness K032340. VISTA$\mathrm{KON}^{\circledR}$ (galyfilcon A) Contact Lens. Available from: https://www. accessdata.fda.gov/cdrh_docs/pdf3/K032340.pdf. Accessed March 14, 2018.

38. FDA 510(k) Summary of Safety and Effectiveness K042275. VISTAKON ${ }^{\circledR}$ (senofilcon A) Soft Contact Lens. Available from: https://www. accessdata.fda.gov/cdrh_docs/pdf4/k042275.pdf. Accessed March 14, 2018.

39. FDA 510(k) Summary K131208. Bausch + Lomb samfilcon A Contact Lens. Available from: https://www.accessdata.fda.gov/cdrh_docs/ pdf13/K131208.pdf. Accessed March 14, 2018.

40. Wygladacz K, Hook D, Steffen R, Reindel W. Breaking the cycle of discomfort. CL Spectrum. 2014;29(13):23-28.

41. Hoteling AJ, Nichols WF, Harmon PS, et al. Characterization and quantitation of PVP content in a silicone hydrogel contact lens produced by dual-phase polymerization processing. J Biomed Mater Res B Appl Biomater. 2018;106(3):1064-1072.

42. ISO Standard 18369-4:2017. Ophthalmic optics - Contact lenses Part 4: Physicochemical properties of contact lens materials. Geneva: International Organization for Standardization; 2017.

43. Thevisioncouncil.org. Eyes overexposed: the digital device dilemma. 2016 Digital Eye Strain Report. Alexandria, VA: The Vision Council. 2016 [cited September 1, 2017]. Available from: https://visionimpactinstitute.org/wp-content/uploads/2016/03/2016EyeStrain_Report_WEB. pdf. Accessed March 14, 2018.

44. Ludington P, Hovinga K, Merchea M. Predicted logMAR image resolution during dehydration for silicone hydrogel contact lenses. Invest Ophthalmol Vis Sci. 2014;55:4636.

45. Mengher LS, Bron AJ, Tonge SR, Gilbert DJ. A non-invasive instrument for clinical assessment of the pre-corneal tear film stability. Curr Eye Res. 1985;4(1):1-7.

46. Torkildsen G. The effects of lubricant eye drops on visual function as measured by the inter-blink interval visual acuity decay test. Clin Ophthalmol. 2009;3:501-506.

47. Gullon M, Schock SE. Soft contact lens visual performance: a multicenter study. Optom Vis Sci. 1991;68(2):96-103.

48. Liu H, Thibos L, Begley CG, Bradley A. Measurement of the time course of optical quality and visual deterioration during tear break-up. Invest Ophthalmol Vis Sci. 2010;51(6):3318-3326.

49. Liu H, Begley C, Chen M, et al. A link between tear instability and hyperosmolarity in dry eye. Invest Ophthalmol Vis Sci. 2009;50(8): 3671-3679.

50. Cheng L, Muller SJ, Radke CJ. Wettability of silicone-hydrogel contact lenses in the presence of tear-film components. Curr Eye Res. 2004; 28(2):93-108.

51. van Beek M, Weeks A, Jones L, Sheardown H. Immobilized hyaluronic acid containing model silicone hydrogels reduce protein adsorption. J Biomater Sci Polym Ed. 2008;19(11):1425-1436.

52. Fonn D, Situ P, Simpson T. Hydrogel lens dehydration and subjective comfort and dryness ratings in symptomatic and asymptomatic contact lens wearers. Optom Vis Sci. 1999;76(10):700-704.

53. Koh S, Maeda N, Hamano T, et al. Effect of internal lubricating agents of disposable soft contact lenses on higher-order aberrations after blinking. Eye Contact Lens. 2008;34(2):100-105.

\section{Dovepress}

PubMed Central and CAS, and is the official journal of The Society of Clinical Ophthalmology (SCO). The manuscript management system is completely online and includes a very quick and fair peer-review system, which is all easy to use. Visit http://www.dovepress.com/ testimonials.php to read real quotes from published authors. 